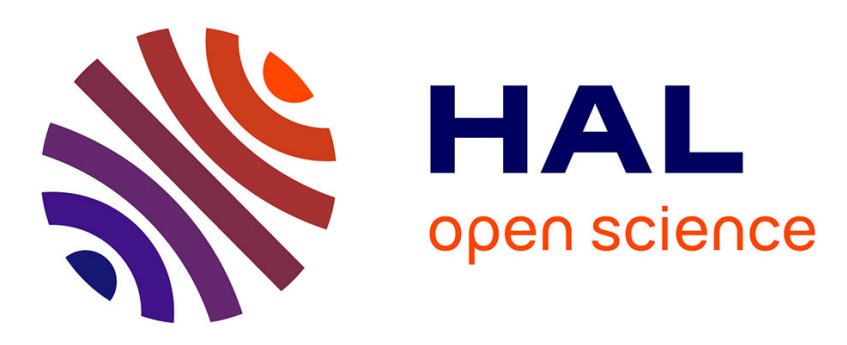

\title{
Take-off and landing of an AWE system using a multicopter
}

Audrey Schanen, Jonathan Dumon, Nacim Meslem, Ahmad Hably

\section{To cite this version:}

Audrey Schanen, Jonathan Dumon, Nacim Meslem, Ahmad Hably. Take-off and landing of an AWE system using a multicopter. ACC 2020 - American Control Conference, Jul 2020, Denver (online),

United States. 10.23919/ACC45564.2020.9148035 . hal-02877182

\section{HAL Id: hal-02877182 \\ https://hal.science/hal-02877182}

Submitted on 22 Jun 2020

HAL is a multi-disciplinary open access archive for the deposit and dissemination of scientific research documents, whether they are published or not. The documents may come from teaching and research institutions in France or abroad, or from public or private research centers.
L'archive ouverte pluridisciplinaire HAL, est destinée au dépôt et à la diffusion de documents scientifiques de niveau recherche, publiés ou non, émanant des établissements d'enseignement et de recherche français ou étrangers, des laboratoires publics ou privés. 


\title{
Take-off and landing of an AWE system using a multicopter
}

\author{
Audrey Schanen, Jonathan Dumon, Nacim Meslem, Ahmad Hably \\ Univ. Grenoble Alpes, CNRS, Grenoble INP*,GIPSA-Lab, \\ F-38000 Grenoble, France. \\ *Institute of Engineering Univ. Grenoble Alpes \\ \{audrey.schanen,jonathan.dumon,nacim.meslem,ahmad.hably\}@gipsa-lab.fr
}

\begin{abstract}
In this paper, the problem of take-off and landing of an airborne wind energy system is addressed. The solution explored is to equipe the airborne wing of the system with a multicopter drone in order to perform the take-off and land maneuvers, even in the absence of wind. The proposed model with the proposed control strategy is implemented and tested in a numerical environment. The results show efficiency of the proposed control law and its robustness with respect to modelling errors and wind gusts.
\end{abstract}

Keywords: Airborne wind energy system, On-ground system, On-board system, nonlinear systems, nonlinear control, flatness, Take-off, landing, tethered drone.

\section{INTRODUCTION}

Airborne wind energy (AWE) systems have attracted a lot of attention in the last few decades (see [1] for a survey on soft kites and [2] for AWE systems in general). AWE is the term given to some innovative flying energy systems that aim at harvesting wind energy at high altitudes using airborne airfoils and tethers. The main idea is to reduce the foundation used for conventional wind turbines in order to reach high altitudes where the wind is assumed to be more stable and stronger. In addition, these energy systems can be installed in a large variety of locations with possibly low costs and more easily implementation on floating platforms.

AWE systems are mainly classified into two categories: Onground and on-board production systems. A ground-based generator/motor is connected to an airborne platform by tethers in the case of on-ground production systems [3], [4]. While for onboard production systems, the wind turbine is mounted on the airborne platform where energy is harvested and sent to ground via conductive tethers [5].

In the literature, modelling, control, and optimization of AWE systems have been extensively studied theoretically [6], in numerical environments [7] and through infield experiments [8][10]. Several innovative designs regarding the airborne platform, its aerodynamic capabilities, and its control have been proposed. Different types of production and retraction trajectories have been studied. Books [11] and [12] and references therein present an in-depth coverage of the latest research and development activities concerning AWE systems.

Despite of the above-mentioned developments of the domain, several relevant and urgent aspects has to be addressed in the way of the technical feasibility and commercial success of AWE systems. One important issue is the take-off and landing of the airborne platform, especially for on-ground production systems with rigid airfoils. In [13], a control-based approach has been proposed to a rotational take-off of an AWE system. In the Airborne
Wind Energy Conference (AWEC 2017), [14] has presented three approaches for launching and landing of an EnerKite wing [15]: Vertical take-off, catapult, and rotating arm. In [16], a theoretical analysis of different concepts for only the take-off phase of AWE systems based on rigid wings and on-ground power conversion has been presented. A deeper numerical simulation of a linear take-off maneuver combined with on-board propellers has been also studied. The limitation of this maneuver is that it cannot be used for the landing phase in addition to the impacts on the ground station design.

For flexible wing/kite AWE systems, strategies such as static mast-based launch and landing used by [17] or a rotating arm concept used by Kitegen [18], have been explored. The problem of automatically retracting the wing of an AWE system during the reel-in phase has been addressed in [19]. In [20], static and dynamic feasibility analyses of using multicopter for autonomously launching and landing kites have been carried out. The authors pointed out a disadvantage in this solution for soft kites that is the kite generates a lift force opposite to the propeller thrust of the multicopter during the hovering phase. To remedy this problem, in a recent work [21], a vertical take-off strategy, using a multicopter, of a flexible kite AWE system have been studied in a simulation environment. The landing of the kite is done by the winch of the on-ground station without the multicopter assistance.

In this paper, we will present a solution for the problem of takeoff and landing of a rigid-wing airborne wind energy system using a multicopter. The forces generated by the multicopter are added to the forces of the AWE model. In addition, we will present a control strategy to fulfill this objective. The paper is organized as follows. The system modeling is given in section II. The controller design is addressed in section III. The numerical simulation conditions are given in section IV followed by a discussion of the results in section V. The paper ends by conclusions and future work in section VI. The novel contribution stands in the application of this kind of control strategy to this kind of AWE system.

\section{System Modeling}

As mentioned in the introduction, the airborne wind energy (AWE) system studied in this present paper is composed of two elements: The first element is a flying device composed of a rigid wing with a multicopter or a drone and the second one is an on-ground station. Both elements are connected through a tether (Fig.1). In presence of wind, the device generates aerodynamic lift and drag forces. In the case of on-ground systems, the resultant traction force is transferred via the tether to the on-ground 
generator where a drum is used to convert the linear motion of the tether into shaft power, which is then used to drive a generator. The role of the drone attached to the flying device is to create a thrust force. Those forces related to the drone are added to the model presented in [22]. The study is presented in $2 \mathrm{D}$, to simplify the system and the controller design. This simplification can be made since another controller can be implemented to align the front of the drone to the on-ground generator. As long as the front of the drone points to the origin, the system can be express in 2D without loosing informations. In order to find the

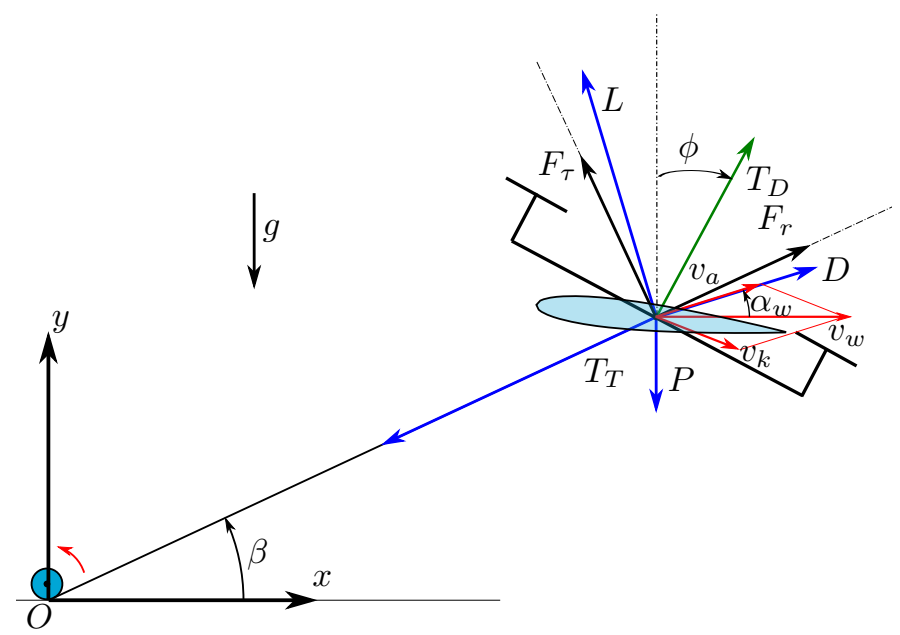

Fig. 1. The airborne wind energy system with the drone connected to the on-ground station. All forces acting on the system are shown.

model, one has to express all the forces acting on the system. Firstly, we will consider the aerodynamic forces generated by the wing in presence of wind. They depend on the profile and aerodynamic properties of the wing. So, the lift and drag forces can be expressed by:

$$
L=\frac{1}{2} \rho S v_{a}^{2} C_{L}, \quad D=\frac{1}{2} \rho S v_{a}^{2} C_{D}
$$

where $\rho$ is the air density, $v_{a}$ is the apparent wind velocity, $S$ is the considered wing surface, $C_{L}$ and $C_{D}$ are respectively aerodynamic lift and drag coefficients. One can use the curves of Fig. 2, designed partially for example from equations of [23] in order to determine these parameters. As it is shown on Fig. 1, $D$ is aligned in the direction of apparent wind $v_{a}$ and $L$ is orthogonal to it. The angle of attack will be defined here as the sum of the pitch angle $\alpha_{u}$ determined with respect to the horizon and the relative wind orientation $\alpha_{w}$.

The apparent wind velocity $\vec{v}_{a}$ is defined by:

$$
\vec{v}_{a}=\vec{v}_{w}-\vec{v}_{k}
$$

where $\vec{v}_{k}$ is the translation velocity of the flying device and $\vec{v}_{w}$ the wind speed. In this present study, the movement of the system is assumed to be in the vertical plane. It is also assumed that the tether of length $r$ is always taut and forms a straight line. This assumption is available for small tether's length since in this case the linear mass is negligible. Otherwise, a tether model should be add in order to take into account its influence. The tension in the tether is $T_{T}$. The tether has an elevation angle $\beta$ with respect to the ground plane. The drone has an inclination angle $\phi$ with respect to the vertical axis and produces a thrust force $T_{D}$. The

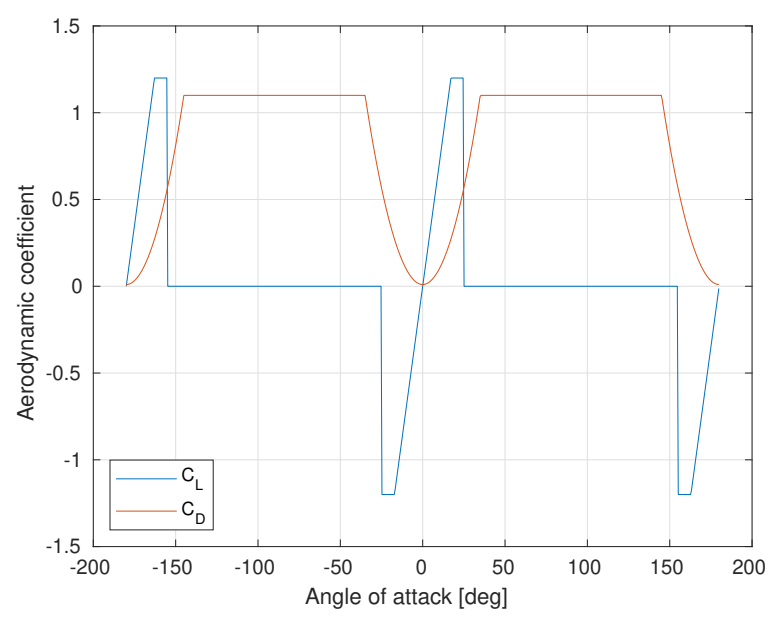

Fig. 2. Aerodynamic coefficient $C_{L}$ and $C_{D}$ in function of the wing's angle of attack.

combined mass of all airborne system components is denoted by $M_{M}$. For the drone attached to the rigid wing, its closed-loop dynamics model can be expressed by the following equation:

$$
\dot{T}_{D}=\frac{1}{\tau_{T_{D}}}\left(T_{D}^{d}-T_{D}\right), \quad \dot{\phi}=\frac{1}{\tau_{\phi}}\left(\phi^{d}-\phi\right)
$$

where $T_{D}^{d}$ and $\phi^{d}$ are the desired thrust and inclination's angle. $\tau_{T_{D}}$ and $\tau_{\phi}$ the time constants of the first order systems of eq. 3 .

Fundamental dynamics principles are used to establish the dynamic model. Considering the system's two degrees of freedom, $r$ and $\beta$, translation velocity of the flying device $\vec{v}_{k}$ can be decomposed into a radial velocity component $v_{k, r}=\dot{r}$ and a tangential velocity component $v_{k, \tau}=r \dot{\beta}$. As done in the authors' previous work [22], differentiation of $\vec{v}_{k}$ with respect to time yields a radial acceleration component and a tangential acceleration component:

$$
\frac{d v_{k, r}}{d t}=\ddot{r}-r \dot{\beta}^{2}, \quad \frac{d v_{k, \tau}}{d t}=r \ddot{\beta}+2 \dot{r} \dot{\beta}
$$

The resultant forces $F_{r}$ and $F_{\tau}$ on the system are respectively the radial and tangential force components according to the polar coordinate system $(r, \beta)$ as shown on Fig. 1 .

$$
\begin{aligned}
F_{r}= & -T+L \sin \left(\beta-\alpha_{w}\right)+D \cos \left(\beta-\alpha_{w}\right) \\
& -P \sin \beta-T_{D} \sin (\phi-\beta) \\
F_{\tau}= & L \cos \left(\beta-\alpha_{w}\right)-D \sin \left(\beta-\alpha_{w}\right) \\
& -P \cos \beta+T_{D} \cos (\phi-\beta)
\end{aligned}
$$

where $T$ is the effective torque of the drum divided by its radius $R_{d}$. The tension of the tether is then calculated as follows: $T_{T}=$ $T+M_{D} \ddot{r} . P$ is the weight of the system. $\alpha_{w}$ is the angle that the apparent wind velocity forms with the horizontal. It can be calculated with the formula of [23].

$$
\alpha_{w}=\arctan \frac{r \cos (\beta) \dot{\beta}+\dot{r} \sin (\beta)}{v_{w}+r \sin (\beta) \dot{\beta}-\dot{r} \cos (\beta)}
$$

The dynamic model can then be derived in $2 \mathrm{D}$ polar coordinates:

$$
\begin{aligned}
\ddot{\beta} & =\frac{1}{r}\left[-2 \dot{\beta} \dot{r}+\frac{F_{\tau}}{M_{M}}\right] \\
\ddot{r} & =\frac{1}{M_{M}+M_{D}}\left[r \dot{\beta}^{2} M_{M}+F_{r}\right]
\end{aligned}
$$


where $M_{D}=\frac{I}{R_{d}^{2}}$ is expressed in function of the moment of inertia of the on-ground generator $I$ and its radius $R_{d}$. In addition to these equations, one adds the dynamic equation of the onground generator traction force:

$$
\dot{T}=\frac{1}{\tau_{T}}\left(u_{r}^{d}-T\right)
$$

where $u_{r}^{d}$ is the desired traction force and $\tau_{T}$ is the time constant of the dynamic system modeled in this present paper by a first order dynamic system.

To summarize, the overall system's nonlinear model can be written as:

$$
\begin{aligned}
\ddot{\beta}= & \frac{1}{r}\left[-2 \dot{\beta} \dot{r}+\frac{1}{M_{M}}\left(-P \cos (\beta)+u_{\beta}\right.\right. \\
& \left.\left.+L \cos \left(\beta-\alpha_{w}\right)-D \sin \left(\beta-\alpha_{w}\right)\right)\right] \\
\ddot{r}= & \frac{1}{M_{M}+M_{D}}\left[r \dot{\beta}^{2} M_{M}-T-P \sin (\beta)+u_{T_{0}}\right. \\
& \left.+L \sin \left(\beta-\alpha_{w}\right)+D \cos \left(\beta-\alpha_{w}\right)\right]
\end{aligned}
$$

where the system's inputs are $u_{r}^{d}, u_{\beta}:=T_{D} \cos (\phi-\beta)$ and $u_{T_{0}}:=-T_{D} \sin (\phi-\beta)$. In this present paper, for sake of control design and without any loss of generality, the lift and drag forces are considered as disturbances. Thus, the control law has to ensure the desired performance and in the same time to compensate the effect of the poorly-known lift and drag forces.

\section{Controller Design}

In this section, we will consider the design of $u_{r}^{d}, u_{T_{0}}$ and $u_{\beta}$. An output feedback linearization method presented in the literature is used. Based on the flatness analysis, at the first stage, an output-feedback linearization control is computed. The aim of this stage is to transform the nonlinear tracking problem to a simple stabilizing problem. Then, at the second stage, an intermediate linear control law is computed to ensure the asymptotic stability of the tracking error which is now linear. For this part, the considered input of the system is $u=\left(\begin{array}{l}u_{r}^{d} \\ u_{\beta}\end{array}\right)$, the considered state variables are $r, \dot{r}, \beta$ and $\dot{\beta}$, and the considered output is $y=\left(\begin{array}{l}r \\ \beta\end{array}\right)$. The states $r, \dot{r}, \beta$ and $\dot{\beta}$ can be measured using some sensors as explained in [22]. For the control design, the dynamic of $T$ is neglected, so $T=u_{r}^{d}$. Then, the following simplified model is considered to design the controller:

$$
\dot{x}=\left(\begin{array}{c}
\dot{r} \\
\frac{1}{M_{M}+M_{D}}\left[r \dot{\beta}^{2} M_{M}+\left(-u_{r}^{d}-P \sin (\beta)+u_{T_{0}}\right)\right] \\
\dot{\beta} \\
\frac{1}{r}\left[-2 \dot{\beta} \dot{r}+\frac{1}{M_{M}}\left(-P \cos (\beta)+u_{\beta}\right)\right]
\end{array}\right)
$$

with the state variable $x=\left(\begin{array}{llll}r & \dot{r} & \beta & \dot{\beta}\end{array}\right)^{T}$. As it can be clearly shown, the relative degree of the system is 2 . This corresponds to the number of times one needs to differentiate the output $y$ before the input $u$ appears explicitly (see eq. 14).

$$
\begin{gathered}
y=\left(\begin{array}{cccc}
1 & 0 & 0 & 0 \\
0 & 0 & 1 & 0
\end{array}\right) x, \quad \dot{y}=\left(\begin{array}{llll}
0 & 1 & 0 & 0 \\
0 & 0 & 0 & 1
\end{array}\right) x \\
\dot{y}=\left(\begin{array}{c}
\dot{r} \\
\dot{\beta}
\end{array}\right)=\left(\begin{array}{llll}
0 & 1 & 0 & 0 \\
0 & 0 & 0 & 1
\end{array}\right) x
\end{gathered}
$$

$$
\begin{gathered}
\ddot{y}=\underbrace{\left(\begin{array}{c}
\frac{1}{M_{M}+M_{D}}\left[r \dot{\beta}^{2} M_{M}-P \sin (\beta)+u_{T_{0}}\right] \\
\frac{1}{r}\left[-2 \dot{\beta} \dot{r}-\frac{P}{M_{M}} \cos (\beta)\right]
\end{array}\right)}_{b(x)} \\
+\underbrace{\left(\begin{array}{cc}
-\frac{1}{M_{M}+M_{D}} & 0 \\
0 & \frac{1}{M_{M}} \frac{1}{r}
\end{array}\right)}_{A(x)} \underbrace{\left(\begin{array}{l}
u_{r}^{d} \\
u_{\beta}
\end{array}\right)}_{u}
\end{gathered}
$$

From eq. (14), one gets that $\ddot{y}$ is affine with respect to the input $u$. Under the hypothesis that the matrix $A(x)$ is invertible, which is the case in our operating range, the nonlinear control law defined by:

$$
u=A^{-1}(x)(v-b(x))
$$

reduces the nonlinear system's dynamics to the dynamics of a double integrator $\ddot{y}=v$ with $v$ is the linear intermediate control law. Thus, to ensure the stability of the tracking error, we propose to use a multi-variable proportional-integral-derivative controller, $v=\left(\begin{array}{c}v_{r} \\ v_{\beta}\end{array}\right)$ with

$$
v_{r}=\alpha_{r_{-1}} \int_{0}^{t} e_{r}(\tau) \mathrm{d} \tau+\alpha_{r 0} e_{r}+\alpha_{r 1} \dot{e}_{r}+\ddot{y}_{r}^{d}
$$

$$
v_{\beta}=\alpha_{\beta_{-1}} \int_{0}^{t} e_{\beta}(\tau) \mathrm{d} \tau+\alpha_{\beta 0} e_{\beta}+\alpha_{\beta 1} \dot{e}_{\beta}+\ddot{y}_{\beta}^{d}
$$

where $y^{d}=\left[\begin{array}{ll}y_{r}^{d} & y_{\beta}^{d}\end{array}\right]^{T}$ is the desired output reference, $e=$ $\left(y^{d}-y\right)$ is the tracking error. Note that, the integral term is added in order to reject disturbances and to compensate modelling errors, for instance the non considered lift and drag forces. This integral term assures that the model is robust. The Integral of the Time weighted Absolute Error (ITAE) performance index is used in order to tune the controller parameters. It allows to specify the dynamic response with relatively small overshoot and relatively little oscillation. The used coefficients are $\left(\begin{array}{llll}1 & 1.75 w_{n} & 2.15 w_{n}^{2} & w_{n}{ }^{3}\end{array}\right)$ with $w_{n}$ the natural frequency of the closed-loop system. Note that, by this choice one can get a stable linear tracking error with a desired converging rate.

For the design of $u_{T_{0}}$, the control law can be obtained by using the following static equation:

$$
u_{T_{0}}=\underbrace{T_{0}^{d}+M_{M} \ddot{r}-r \dot{\beta}^{2} M_{M}+P \sin (\beta)}_{u_{T_{0}}^{d}}+\underbrace{T_{0}^{d}-T_{T}}_{\Delta u_{T_{0}}}
$$

where $u_{T_{0}}^{d}$ is the desired tension of the tether and $\Delta u_{T_{0}}$ is the error on the tension due to disturbances, like lift and drag. This control law corresponds to a feed-forward and allows to correct modelling errors.

By decomposing the action of the drone into a radial component and tangential one, it is important to mention that the control variable of the elevation angle and the radial part enters directly in concurrence with the winch's torque. One has a system of 2 actuators, $T$ and $u_{T_{0}}$ in order to control 2 variables: $r$ and $T$. Choosing to directly control $T$ with $T^{d}$, while controlling the winch torque will allow to control precisely the tension in the tether. On the other hand, to control $r$, one has to use the thrust generated by the drone. That is why we choose to use $T$ 
to control $r$, which is a more critical variable of the system than tether tension that just has to be maintained positive. The block diagram on Fig. 3, shows the different stages to be carried out to provide the correct control action. The Drone block corresponds to a simplified model presented in eq.3. The Wing block is the model given by eq. 1 and Fig. 2 .

\section{Simulation Conditions}

In this section, different parameters used in the simulation environment are presented. In Table I, we give the values of the physical variables of the system. As it can be noticed, we have used values similar to those used in our previous work [22] since our objective in the near future is to validate experimentally the control design at Gipsa-lab's indoor wind tunnel. This also justifies the choice of different time constants and upper and lower bounds on the tension in the winch as well as the bounds on the drone's thrust and its inclination angle. Actuators $T, T_{D}$ and $\phi$ are saturated between respectively $T_{\min }$ and $T_{\max }, T_{D_{\min }}$ and $T_{D_{\max }}$, and $\phi_{\min }$ and $\phi_{\max }$. At the end of Table I, the values of the natural frequency are given. Our objective is to decouple the dynamics of the tether's tension from the dynamics of the elevation angle. In Table II, the parameters of the simulation scenario are given. The simulation starts towards the vertical above the ground as it can be noticed from the values of $r_{0}$ and $\beta_{0}$. The desired tension in the tether is equal to $1 \mathrm{~N}$ in order to keep the tether taut.

TABLE I

PHySicAl AND CONTROLler PARAMETERS

\begin{tabular}{|l|l|l|}
\hline Symbol & Name & Value \\
\hline$M_{M}$ & Mass of airborne subsystem & $0.11 \mathrm{~kg}$ \\
$M_{D}$ & Ground station rotor mass & $0.0481 \mathrm{~kg}$ \\
$S$ & Wing area & $0.14 \mathrm{~m}^{2}$ \\
$\rho$ & Air density & $1.225 \mathrm{~kg} / \mathrm{m}^{3}$ \\
$\tau_{T}$ & Time constant of motor current loop & $0.07 \mathrm{~s}$ \\
$\tau_{T_{D}}$ & Time constant of drone thrust loop & $0.1 \mathrm{~s}$ \\
$\tau_{\phi}$ & Time constant of drone inclination loop & $0.2 \mathrm{~s}$ \\
$T_{\min }$ & Minimum tension in the winch & $-2 \mathrm{~N}$ \\
$T_{\max }$ & Maximum tension in the winch & $2 \mathrm{~N}$ \\
$T_{D \min }$ & Minimum drone's thrust & $0 \mathrm{~N}$ \\
$T_{D \max }$ & Maximum drone's thrust & $3 \mathrm{~N}$ \\
$\phi_{\min }$ & Minimum drone's inclination angle & $-50 \mathrm{deg}$ \\
$\phi_{\max }$ & Maximum drone's inclination angle & $50 \mathrm{deg}$ \\
$\omega_{n r}$ & Natural frequency for the tension loop & $3 \mathrm{rad} / \mathrm{s}$ \\
$\omega_{n \beta}$ & Natural frequency for $\beta$ loop & $1.5 \mathrm{rad} / \mathrm{s}$ \\
\hline
\end{tabular}

TABLE II

PARAMETERS OF THE SIMULATION'S SCENARIO

\begin{tabular}{|l|l|l|}
\hline Symbol & Name & Value \\
\hline$r_{0}$ & Initial tether's length & $0.2 \mathrm{~m}$ \\
$\beta_{0}$ & Initial elevation angle & $90 \mathrm{deg}$ \\
$T_{D_{0}}$ & Initial drone's thrust & $0 \mathrm{~N}$ \\
$\phi_{0}$ & Initial drone's inclination angle & $0 \mathrm{deg}$ \\
$r_{f}$ & Final tether's length & $5 \mathrm{~m}$ \\
$\beta_{f}$ & Final elevation angle & $60 \mathrm{deg}$ \\
$T_{0}^{d}$ & Desired tension in the tether & $1 \mathrm{~N}$ \\
$\alpha_{u}$ & Wing's pitch angle & $0 \mathrm{deg}$ \\
\hline
\end{tabular}

The simulation scenario is divided into different phases:

- Initial phase: In this phase, the system starts from its initial position at $r_{0}, \beta_{0}, T_{D_{0}}$ and $\phi_{0}$.
- Take-off phase: At time $t=30 \mathrm{~s}$ the reference signal $r^{d}$ goes to $r_{f}$ with a ramp rate of $0.33 \mathrm{~m} / \mathrm{s}$, and the desired elevation angle $\beta^{d}$ goes to $\beta_{f}$ with a ramp rate of $4 \mathrm{deg} / \mathrm{s}$.

- Landing phase: At time $t=60 \mathrm{~s}$ the reference signal $r^{d}$ goes to $r_{0}$ with a ramp rate of $0.33 \mathrm{~m} / \mathrm{s}$ and $\beta^{d}$ goes to $\beta_{0}$ with a ramp rate of $4 \mathrm{deg} / \mathrm{s}$.

Two simulation tests are considered, the first one with no wind and the second one with a constant wind of $2 \mathrm{~m} / \mathrm{s}$. To be close to the real world case, in the simulation tests, we have added measurement noise on all the state variables. This allows to validate the proposed control approach, where the sensor's data are not perfect.

\section{Results And Discussion}

In this section, the simulation results are presented and discussed. Figure 4 shows the trajectory followed by the drone with the desired path. The drone has succeeded to reached each point as requested in the simulation scenario presented at the end of Section IV. One can note that there is also an overshoot on tether length $r$, especially at the end of the landing phase, that has to be carefully designed as it can lead to a crash of the system. Other variables of interest of the simulation are shown on Figs. 5- 8 . Figure 5 shows that the system succeed to reel-out and reelin the tether following the desired reference, with a reasonable overshoot of $2 \%$. Figure 5 also shows that the elevation angle $\beta$ loop stabilizes at the desired position with an overshoot of $2 \%$. Differences between simulated and desired value is due to the first order model of the actuator used to model the current loop of the winch's driver. This smooths the overshoots of the control, but it does not introduce any delay, since the control dynamics is slower than that of the actuator.

The tether tension $T_{T}$, as shown on Fig. 6, is always positive meaning that the tether is always taut. Moreover, the tether tension is close to the desired value $T_{0}^{d}$, showing the efficiency of the control loop.

Regarding $T_{D}$ and $\phi$ shown on Fig. 7, desired values are directly calculated from $u_{\beta}$ and $u_{T_{0}}$. The saturation on the angle $\phi$ and $T_{D}$ are not reached and dynamics of $T_{D}$ and $\phi$ that smooth overshoots on desired variables manage to follow reference signals without introducing a significant delay. This shows that dynamics of $\beta$ and $r$ control loops have been correctly tuned regarding to the drone capacity.

As mentioned in previous sections, the drone thrust can be disturbed by the aerodynamic forces and be saturated beyond wind values. It also has a slower dynamics than that of the current loop of the winch. It is therefore more efficient to control $r$ with the fastest actuator and the least sensitive to disturbances, using the thrust of the drone and eventually the aerodynamic forces of the wing in the case of a control of the angle of pitch. The idea is to control the tension of the tether in order to maintain it constant or to maximize it when the system is in the energy production phase as it has been done in [23].

Lift and drag forces, computed from apparent wind, and aerodynamics coefficients are shown on Fig. 8. $C_{L}$ and $C_{D}$ coefficients are calculated from $\alpha_{w}$ and the model presented on Fig. 2. Since there is no wind, variations on $L$ and $D$ only occurs when there is a variation of $\beta$ or $r$. For the test with the wind at $2 \mathrm{~m} / \mathrm{s}$, figures for the trajectory and evolution of $r$ and $\beta$ are shown. Figure 9 shows the trajectory followed by the drone with the desired path. The influence of the wind can be seen mostly 


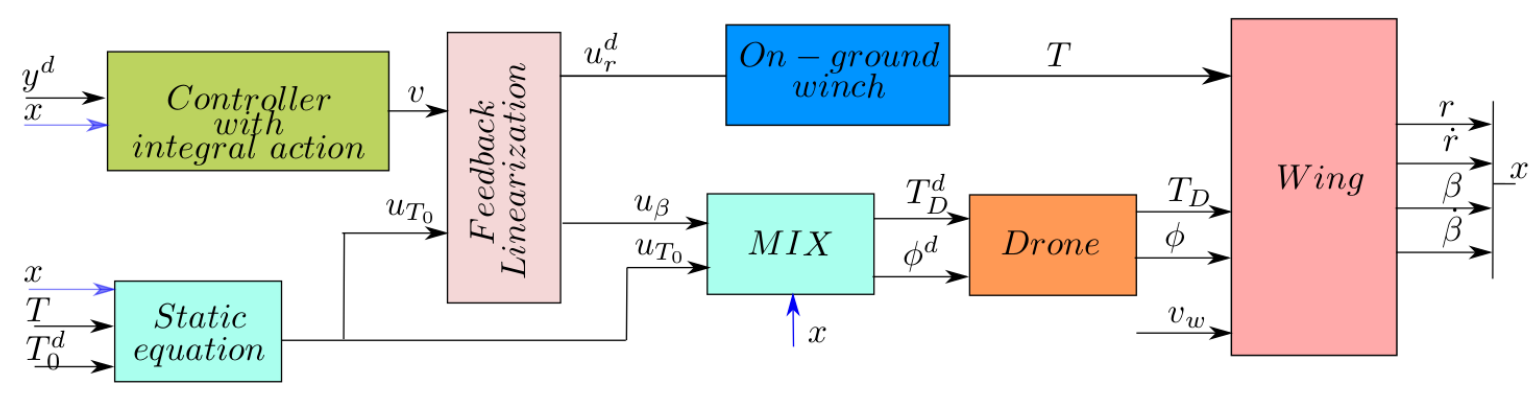

Fig. 3. Block diagram of the control strategy.

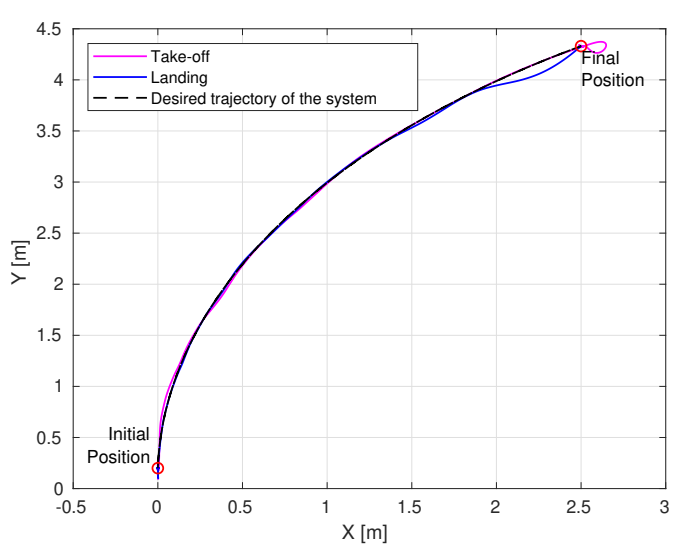

Fig. 4. Trajectory of the drone and the desired path.
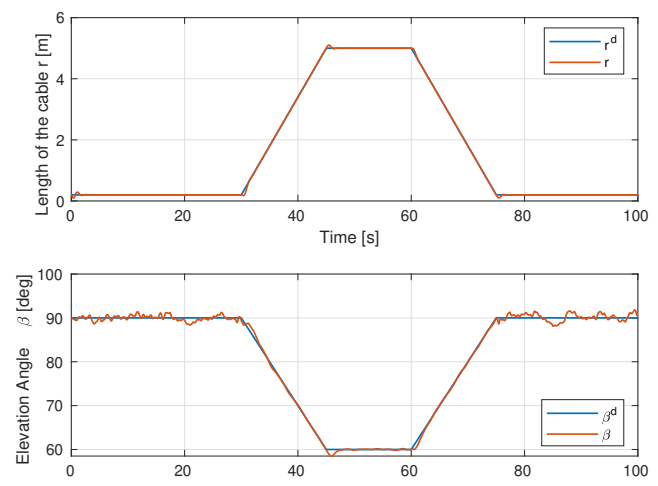

Fig. 5. Evolution of tether's length $r$ and elevation angle $\beta$ compared to their desired values.

on the take-off where it oscillates more around the desired path. Figure 10 shows that the wind has a small impact on the tether's length $r$ regulation but the elevation angle $\beta$ is more disturbed by the wind. However, the proposed control strategy succeeds to stabilize the system at the desired target position even in the presence of wind. As aerodynamic forces are function of the square of the apparent wind, $\mathrm{L}$ and $\mathrm{D}$ are even more sensitive to $\beta$ and $r$ as wind goes stronger. This justifies the coupling between the variables $\beta, r$ and $T$. A control loop can be eventually added on $L$ and $D$, by means of the pitch angle of the wing. In this present paper, the pitch angle has been maintained at 0 during the whole simulation scenario. This opens some perspectives for a
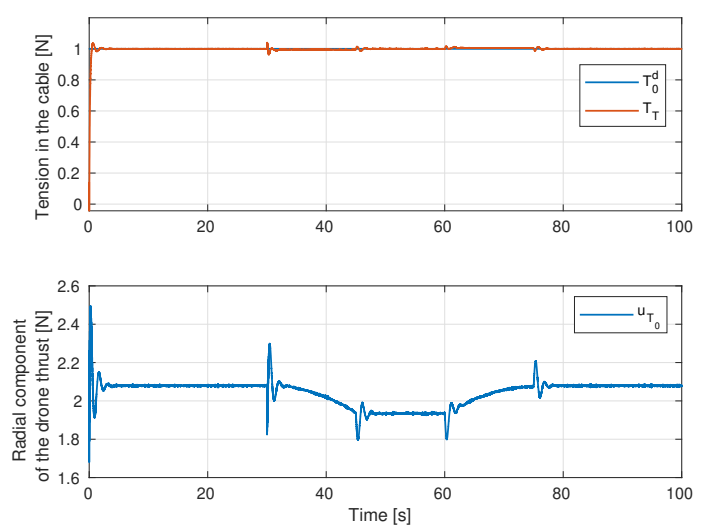

Fig. 6. Evolution of the tension $T_{T}$ compared to its desired value and its control input $u_{T_{0}}$.
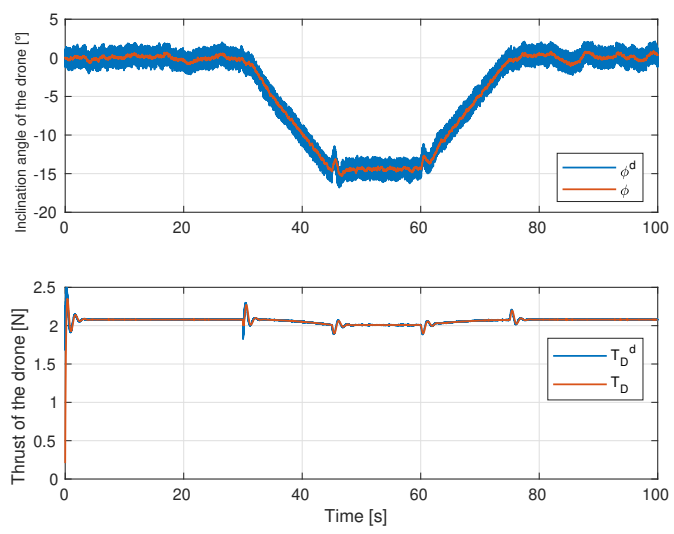

Fig. 7. Evolution of the drone variables $T_{D}, \phi$ compared to their desired values.

future work. On the other hand, the control of $T_{0}$ is principally designed to keep the tether taut. As the wind increases, the force demanded by the drone to keep the tether tension at $T_{0}$ will decrease to 0 . At this stage, there will be enough wind to stretch the tether without the need of the drone that will be stopped. The tension in the tether will no longer necessarily be maintained at $T_{0}$, but may take larger values. 

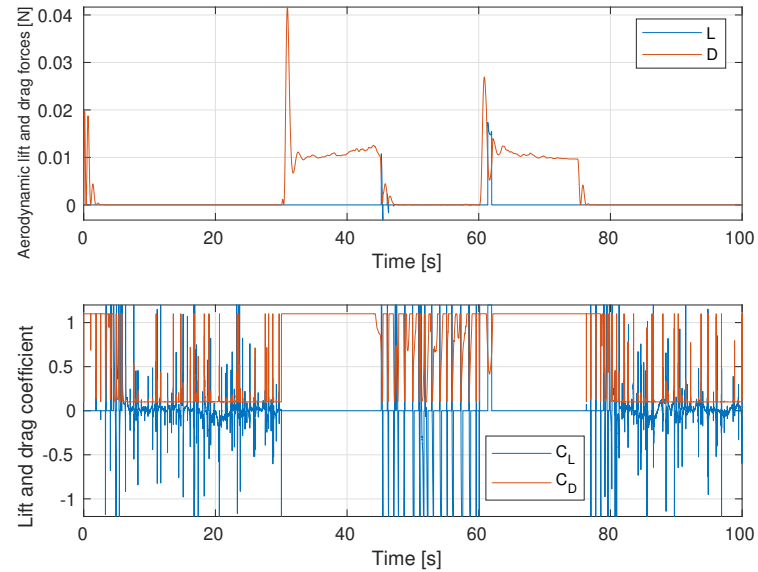

Fig. 8. Evolution of the aerodynamic forces $L$ and $D$ and the corresponding coefficient $C_{L}$ and $C_{D}$.

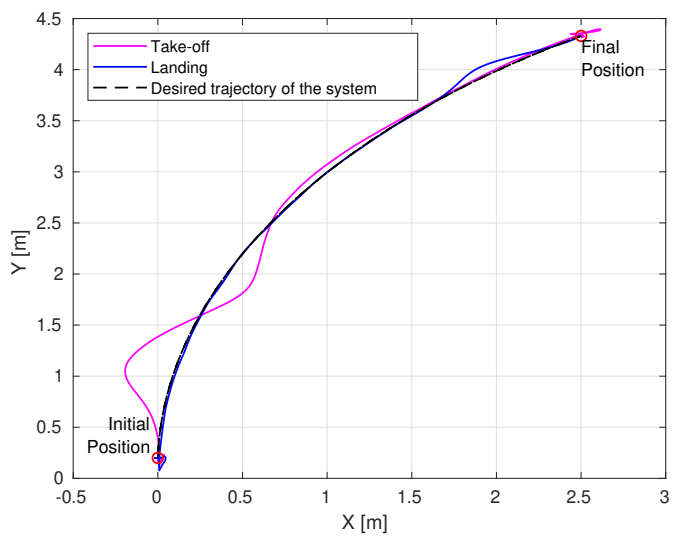

Fig. 9. Trajectory of the drone and the desired path.
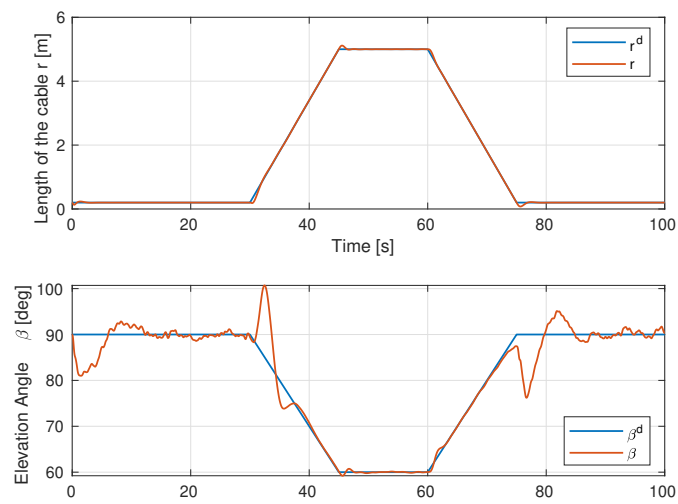

Fig. 10. Evolution of tether's length $r$ and elevation angle $\beta$ compared to their desired values.

\section{CONCLUSION}

In this work, we have presented the modelling and control in simulation environment of a hybrid tethered system that is composed of a rigid wing attached to multicopter drone both attached to the on-ground winch that we control its torque. This system presents a typical example of an AWE system with an aerodynamic profile to harvest the wind energy attached to a drone that has the ability to vertically take-off and land the whole system especially in absence of wind. The control strategy used in our previous work for energy production cycles of AWE systems can also be used for the take-off and landing phases, especially in absence of wind. In the near future, we are working on the control design to take into account the coupling between the tether length $r$, its tension $T_{T}$, its inclination angle $\beta$, the drone and the aerodynamic forces of the wing. Experimental implementation with a small scale prototype are also planned. The mix block will also be improved to take into account the influence of the wing.

\section{REFERENCES}

[1] M. Ahmed, A. Hably, and S. Bacha, "High altitude wind power systems: A survey on flexible power kites," in 2012 XXth International Conference on Electrical Machines, Sept 2012, pp. 2085-2091.

[2] A. Cherubini, A. Papini, R. Vertechy, and M. Fontana, "Airborne wind energy systems: A review of the technologies," Renewable and Sustainable Energy Reviews, vol. 51, pp. 1461-1476, 2015.

[3] L. Fagiano, A. U. Zgraggen, M. Morari, and M. Khammash, "Automatic crosswind flight of tethered wings for airborne wind energy:modeling, control design and experimental results," IEEE Transactions on Control System Technology, vol. 22, no. 4, pp. 1433-1447, July 2014.

[4] M. Erhard and H. Strauch, "Flight control of tethered kites in autonomous pumping cycles for airborne wind energy," Control Engineering Practice, vol. 40, pp. 13-26, July 2015.

[5] Makani Power. [Online]. Available: http://www.makanipower.com

[6] I. Argatov and R. Silvennoinen, "Asymptotic modeling of unconstrained control of a tethered power kite moving along a given closed-loop spherical trajectory," Journal of Engineering Mathematics, vol. 72, no. 1, pp. 187-203, 2012.

[7] Y. Gupta, J. Dumon, and A. Hably, "Modeling and control of a magnus effect-based airborne wind energy system in crosswind maneuvers," IFAC-PapersOnLine, vol. 50, no. 1, pp. 13 878-13 885, 2017.

[8] Kite Power Solutions Ltd. [Online]. Available: http://www.kitepowersolutions.com/

[9] TwingTec. [Online]. Available: http://twingtec.ch

[10] KiteMill. [Online]. Available: http://www.kitemill.com

[11] U. Ahrens, M. Diehl, and R. Schmehl, Eds., Airborne Wind Energy, ser. Green Energy and Technology. Springer, 2013.

[12] R. Schmehl, Airborne wind energy: advances in technology development and research. Springer, 2018.

[13] M. Zanon, S. Gros, and M. Diehl, "Rotational start-up of tethered airplanes based on nonlinear mpc and mhe," in 2013 European Control Conference (ECC). IEEE, 2013, pp. 1023-1028.

[14] B. Rieck, M. Ranneberg, A. Candade, A. Bormann et al., "Comparison of launching \& landing approaches," 2017.

[15] EnerKite. [Online]. Available: http://www.enerkite.de/en/

[16] L. Fagiano and S. Schnez, "On the take-off of airborne wind energy systems based on rigid wings," Renewable energy, vol. 107, pp. 473488, 2017.

[17] Skysails. [Online]. Available: https://www.skysails.info

[18] M. Canale, L. Fagiano, and M. Milanese, "Kitegen: A revolution in wind energy generation," Energy, vol. 34, no. 3, pp. 355-361, 2009.

[19] A. U. Zgraggen, L. Fagiano, and M. Morari, "Automatic retraction and full-cycle operation for a class of airborne wind energy generators," IEEE Transactions on Control Systems Technology, vol. 24, no. 2, pp. 594-608, 2016.

[20] F. Bauer, C. M. Hackl, K. Smedley, and R. M. Kennel, "Multicopterbased launching and landing of lift power kites," in Airborne Wind Energy. Springer, 2018, pp. 463-489.

[21] S. Rapp and R. Schmehl, "Vertical takeoff and landing of flexible wing kite power systems," Journal of Guidance, Control, and Dynamics, vol. 41, no. 11, pp. 2386-2400, 2018.

[22] A. Hably, J. Dumon, G. Smith, and P. Bellemain, "Control of a magnus effect-based airborne wind energy system," pp. 277-301, 2018.

[23] R. Lozano, J. Dumon, A. Hably, and M. Alamir, "Energy production control of an experimental kite system in presence of wind gusts," pp. 2452-2459, 2013. 